\title{
Attenuated Total Reflection for Terahertz Modulation, Sensing, Spectroscopy and Imaging Applications: A Review
}

\author{
Yuxin Huang ${ }^{1,2}$, Ranjan Singh ${ }^{3,4}$, Lijuan Xie ${ }^{1,2}$ and Yibin Ying ${ }^{1,2,5, *}$ \\ 1 College of Biosystems Engineering and Food Science, Zhejiang University, 866 Yuhangtang Road, \\ Hangzhou 310058, China; yxhuang@zju.edu.cn (Y.H.); ljxie@zju.edu.cn (L.X.) \\ 2 Key Laboratory of On Site Processing Equipment for Agricultural Products, \\ Ministry of Agriculture and Rural Affairs, Zhejiang University, Hangzhou 310058, China \\ 3 Division of Physics and Applied Physics, School of Physical and Mathematical Sciences, \\ Nanyang Technological University, 21 Nanyang Link, Singapore 637371, Singapore; \\ ranjan.ranjansingh@gmail.com \\ 4 Centre for Disruptive Photonic Technologies, The Photonics Institute, Nanyang Technological University, \\ 50 Nanyang Avenue, Singapore 639798, Singapore \\ 5 Zhejiang A\&F University, 666 Wusu Street, Lin'an, Hangzhou 311300, China \\ * Correspondence: ibeying@zju.edu.cn; Tel.: +86-0571-88982885
}

Received: 3 June 2020; Accepted: 1 July 2020; Published: 8 July 2020

\begin{abstract}
Terahertz (THz) technique has become one of the most promising analytical methods and has been applied in many fields. Attenuated total reflection (ATR) technique applied in THz spectroscopy and imaging has been proven to be superior in functionalities such as modulation, sensing, analyzing, and imaging. Here, we first provide a concise introduction to the principle of ATR, discuss the factors that impact the ATR system, and demonstrate recent advances on $\mathrm{THz}$ wave modulation and THz surface plasmon sensing based on the THz-ATR system. Then, applications on THz-ATR spectroscopy and imaging are reviewed. Towards the later part, the advantages and limitations of THz-ATR are summarized, and prospects of modulation, surface plasmon sensing, spectroscopy and imaging are discussed.
\end{abstract}

Keywords: Terahertz; attenuated total reflection; modulation; sensing; spectroscopy; imaging

\section{Introduction}

Terahertz $(\mathrm{THz})$ radiation generally refers to a band of electromagnetic waves (from 0.1 to $10 \mathrm{THz}$ ) which spans the region between the mid-infrared (MIR) and the microwave [1-3]. In this range, the electromagnetic waves are characterized in properties as follow: The energy levels of photon are quite low (around 1-10 $\mathrm{meV}$ ), and $\mathrm{THz}$ waves are transparent to most dielectric materials while intensely absorbed by water. Besides, most motions like the hydrogen-bonding stretches and torsion, molecular rotations, crystalline phonon vibrations, and low-frequency bond lie in the $\mathrm{THz}$ range [4,5], which means $\mathrm{THz}$ waves are suitable to explore and analyze these motion modes. In the past decades, along with the development of $\mathrm{THz}$ science and technology, both $\mathrm{THz}$ spectroscopy and imaging technique have been widely applied in various fields including material [6,7], communication [8,9], imaging [10-12], biomedicine [13,14], pharmaceutics $[15,16]$, food [17,18], agriculture [19-22], and others, with heated discussions around modulation, sensing, spectroscopy and imaging analysis. THz time-domain spectroscopy (THz-TDS) is very popular in the $\mathrm{THz}$ region, which uses pump and probe pulses to directly measure the electric field. Both amplitude and phase information can be extracted by using fast Fourier transform (FFT), further, the optical 
or dielectric parameters could be calculated $[2,4]$. The interactions between the $\mathrm{THz}$ wave and material have been discussed from various perspectives and the features of the material were obtained by $\mathrm{THz}$ technique, thus making $\mathrm{THz}$ spectroscopy and imaging technique become an emerging characterization method. According to the sample properties and geometrical constraint, three geometries are usually configured in the THz systems: transmission, reflection, and attenuated total reflection (ATR). Transmission geometry is the most common method for samples with moderate absorption. Reflection and ATR geometries are suitable for the strongly absorbing or scattering samples. Restrictions are inevitable for the surface of the sample when using reflection geometry. ATR geometry is advantageous for liquid, powder, or thin film samples without further preparation compared to the others [4].

Research on $\mathrm{THz}$ wave modulation, especially amplitude and polarization, is essential for promoting the rapid applications in $\mathrm{THz}$ spectroscopy and imaging [23]. Modulation of visible and near-infrared light has been easily achieved for decades, while there are still plenty problems to be solved for modulating $\mathrm{THz}$ waves. Modulation devices with metasurfaces, photodoped semiconductors, conductive thin film, many other artificial or natural materials and structures, show drawbacks such as limited bandwidth, poor attenuation ability, and slow switching speed. Many THz polarization controlling components or structures, such as polarization rotators and wave plates, using stacked birefringent slides, dielectric grating, metamaterials, or prism reflections, still exhibit shortcomings like the unexpected thickness of the structures, narrow operational bandwidth, and restricted conditions for large phase differences [24]. Thus, the utilization of ATR geometry in realizing THz wave modulation have been proposed, which has been proved to be highly efficient and easy-to-fabricate and can reach high or multiple modulation performance over a broadband $\mathrm{THz}$ region [23,24].

Surface plasmon resonance (SPR) sensing in the visible and infrared region has been proven to be highly sensitive to the changes in the optical properties of the neighboring media. This sensing method has been widely applied for compounds analysis, especially for biological or chemical molecules [25]. However, in the $\mathrm{THz}$ region, large negative permittivity strongly prohibits electromagnetic fields from penetrating inside a metal [26], thus surface plasmon polariton (SPP) excitation becomes an attractive challenge. An approach aimed at obtaining the SPP at THz frequencies is an urgent need, since it could promote the applications in biochemical spectroscopy, medical imaging, and so forth.

This review summarizes the THz-ATR application in modulation, sensing, spectroscopy and imaging (as shown in Figure 1). The THz attenuated total reflection (THz-ATR) system is first introduced, including the principle and impact factors. Following this, the recent advances on $\mathrm{THz}$ wave modulation and $\mathrm{THz}$ surface plasmon sensing based on this system are demonstrated. Next, the applications in spectroscopy and imaging are reviewed and discussed. Finally, the challenges and outlook of this field are summarized. 


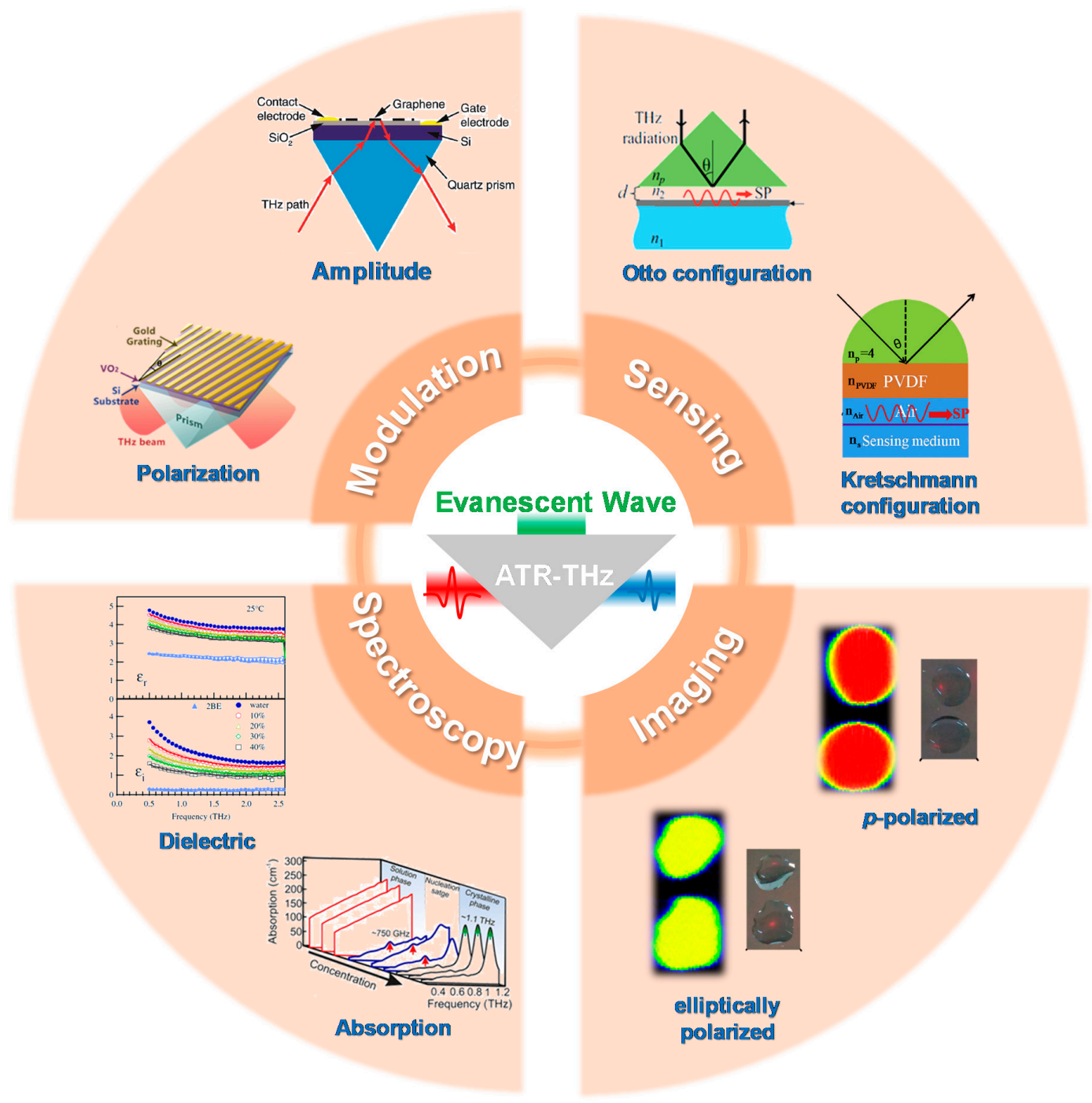

Figure 1. A summary of the THz attenuated total reflection (THz-ATR) application in modulation, sensing, spectroscopy and imaging. Reproduced with permission from [27], Copyright (C 2018, AIP Publishing, [28], Copyright (C) 2016, WILEY-VCH VERLAG GMBH \& CO. KGAA, WEINHEIM, [29], Copyright (C) 2018, Elsevier, [30], Copyright (C) 2012, AIP Publishing, [31], Copyright (C) 2009, Elsevier, [32], Copyright (C) 2016 WILEY-VCH VERLAG GMBH \& CO. KGAA, WEINHEIM, [33], Copyright @ 2017, IOP Publishing.

\section{Theory of ATR}

THz spectroscopy and imaging system, including THz time-domain spectroscopy (THz-TDS), double-modulated differential THz-TDS, THz time-domain imaging (THz-TDI), pulsed THz imaging, continuous-wave $\mathrm{THz}$ imaging, and $\mathrm{THz}$ real-time imaging, were introduced and summarized by Smith et al. [2], Qin et al. [4], Wang et al. [22]. The THz-ATR system consists of basic THz spectroscopy or imaging system and ATR geometry. This system mainly utilizes the properties of evanescent wave resulting from the total internal reflection (TIR). To satisfy the demand of TIR, the ATR crystal should be a high refractive index material. High-resistivity silicon (HR-Si) is the most typical material for ATR crystals with the refractive index of $\sim 3.42$ at the THz region, along with germanium (Ge) with the refractive index of $\sim 4.00$ at the $\mathrm{THz}$ region. The crystal shape, most in the Dove or semicircular prism, depends on the optical geometry in the system. A beam of an electromagnetic wave is refracted as it is illuminated from the side of the ATR crystal at the critical angle, then the wave reflects off the internal surface and thus the evanescent field is generated close to the crystal-sample interface. The optical 
and dielectric parameters can be obtained and extracted through THz-ATR spectroscopy, including refractive index, extinction coefficient, permittivity, dielectric loss, and so forth. The methods of parameters extraction and calculation can be found in the reported research and studies [34]. Choosing the $x z$ plane as the incident plane, the expressions of the evanescent field and the penetration depth are:

$$
\begin{gathered}
\vec{E}_{2}=\vec{A}_{2} \exp \left[-z \frac{2 \pi}{\lambda_{1}} \sqrt{\left.\sin ^{2} \theta_{i}-\frac{n_{2}^{2}}{n_{1}^{2}}\right] \exp \left[i\left(x \frac{2 \pi}{\lambda_{1}} \sin \theta_{i}-\omega t\right)\right],}\right. \\
d_{P}=\frac{n_{1} \lambda_{1}}{2 \pi \sqrt{n_{1}^{2} \sin ^{2} \theta_{i}-n_{2}^{2}}} .
\end{gathered}
$$

while $\vec{A}_{2}$ is the amplitude, $\omega$ is the angular frequency, $t$ is the time, $x$ is the distance in $x$ direction, and $z$ is the distance in $z$ direction. $n_{1}$ is the refractive index of the ATR crystal, $n_{2}$ is the refractive index of the sample. Parameter $\theta_{i}$ is the incident angle, $\lambda_{1}$ is the wavelength of the incident wave. The amplitude of the evanescent wave attenuates exponentially in the $z$ direction. While the penetration depth is usually equal to the order of magnitude of $\lambda_{1}$, which plays an important role on the sensitivity of the ATR method. In the $\mathrm{THz}$ region, the penetration depth $d_{P}$ of the evanescent waves ranges from several micrometers to hundreds of micrometers, which is deeper than that in the infrared region, especially at the low-frequency region. The stability of the THz-ATR system is essential for accurate data acquisition. It shows that the factors such as prism misalignment [35], delay drift [36], incidence angle change [37], total propagation efficiency [38], and thermal expansion [39] could impact the system stability. While, good thermal stability of the system will help minimize the uncertainty of the system [36].

\section{THz Wave Modulation and Devices Based on ATR}

As mentioned before, amplitude and polarization modulation are quite important in $\mathrm{THz}$ spectroscopy and imaging. THz wave modulation through ATR geometry is proven feasible. Compared to the methods such as based on semiconductors, dielectric gratings, birefringent slides, metasurfaces, metamaterials, it is easy to fabricate and achieve high performance or multiple modulation [23,24]. In the following part, these emerging modulation methods and devices based on ATR are introduced in detail.

\subsection{Amplitude Modulation}

Most amplitude modulation devices in the $\mathrm{THz}$ region are based on graphene. This is because graphene, which has ultra-wide spectral response from visible to the $\mathrm{THz}$ frequency range, shows remarkable properties both in optics and electrics. It has high electrical current density, tunable Fermi energy level through optics, electrics, or chemical methods, thus making it a tunable material. The conductivity of graphene could be adjusted by the applied electric field, which would affect the interaction between graphene and the $\mathrm{THz}$ wave. Besides, the ultra-sensitivity to the conductive interface changes from ATR geometry could further amplify these interactions. Thus, making the devices combing graphene and ATR geometry tunable with wider modulation depth, even in the wider range. Significant enhancement of $\mathrm{THz}$ absorption could be realized by a sandwich-structure with monolayer graphene [40]. Through this structure, the interaction between the evanescent wave and graphene layer is controlled by the incidence angle. An extremely large attenuation, up to $\sim 70 \%$ per reflection, was observed especially for s-polarization, and the absorptance shows a proportional relation to the Joule heating amount on the graphene surface. Another approach has been revealed for varied modulation through experimentally fabricating three graphene devices [28]. These devices couple the conductive interfaces with the evanescent waves, thus enhancing the attenuation. The modulation depth of the highly conductive ion-gel graphene (-on-quartz) device (as shown in Figure 2c) can be larger than $90 \%$ at $0.15-0.4 \mathrm{THz}$ and up to $99.3 \%$ at $0.24 \mathrm{THz}$ in s-polarization (as shown in Figure 2b). Results in Figure 2a,b show an obvious voltage dependence from 0.2 to $2 \mathrm{~V}$ due to the conductivity of graphene changed by the voltage. Further, Sun et al. [23] proposed another method 
to enhance $\mathrm{THz}$ modulation, which combines the field confining of metallic gratings and sensitivity of conductive surfaces from the TIR geometry. Modulation at the range of $0.2-1.4 \mathrm{THz}$ could reach $77 \%$. Besides, a THz wave switch with Kretschmann configuration was proposed [41]. This switch consists of a prism with a high refractive index, liquid crystal, and periodically grooved metal grating. The ON-OFF state of the $\mathrm{THz}$ switch was realized by the different reflectivity intensity of the geometrical optics reflection of the $\mathrm{THz}$ wave at the specific value of the liquid crystal refractive index, which was set by the external applied bias voltage. This device showed an extinction ratio of $31.48 \mathrm{~dB}$ at the frequency of $1.0 \mathrm{THz}$. To conclude, deep, tunable, and broad-band amplitude modulation could be realized by combing the materials with tunable optical and dielectric properties and ATR geometry.

(a)
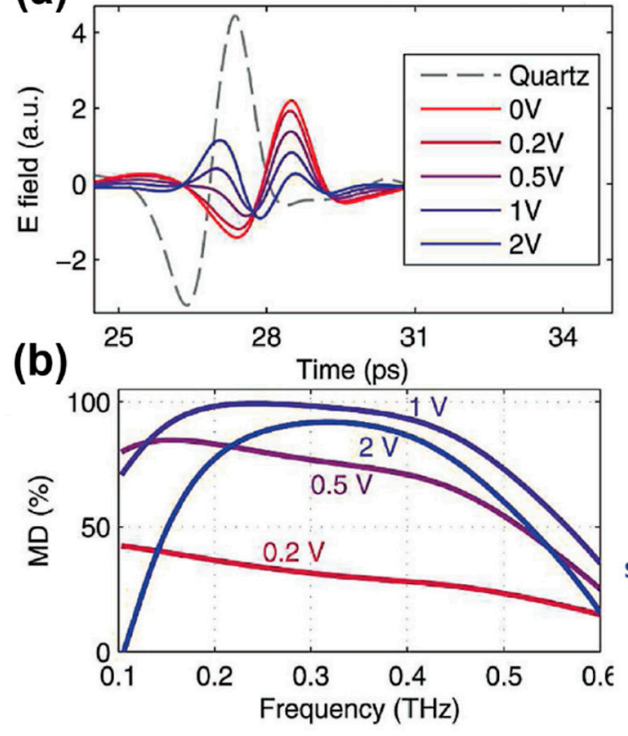

(c)

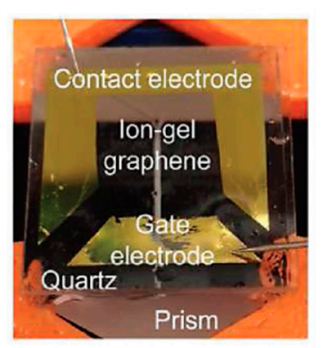

(d)

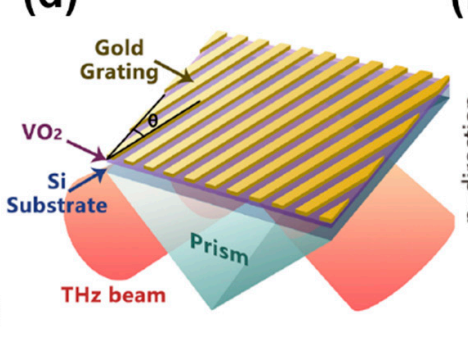

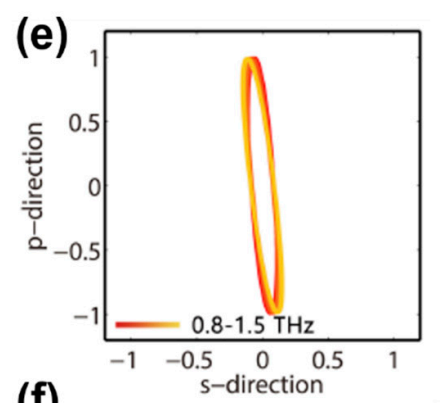

(f)

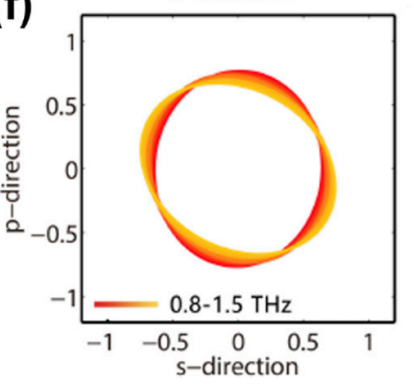

Figure 2. Illustration of the THz wave modulation geometries and devices and results. (a) Time-domain results for the quartz reference and the ion-gel graphene-on-quartz with different voltages. (b) Modulation depth (MD) from 0.1 to $0.6 \mathrm{THz}$ as a function of gate voltage. (c) The ion-gel graphene-on-quartz device. (d) The $\mathrm{VO}_{2}$ device and its combination with the prism. The polarization states from 0.8 to $1.5 \mathrm{THz}$, when the $\mathrm{VO}_{2}$ was in (e) insulating and (f) metallic states, respectively. Reproduced with permission from [28], Copyright (C) 2016, WILEY-VCH VERLAG GMBH \& CO. KGAA, WEINHEIM, [27], Copyright (C) 2018, AIP Publishing.

\subsection{Polarization Modulation}

Similar to the amplitude modulation, high-performance and broad-band and active polarization modulation can be realized with grating structures. Liu et al. 24] proposed a $\mathrm{THz}$ polarization converter operating in TIR geometry with a metal wire grating, which can achieve three functions, including achromatic $45^{\circ}$ polarization rotation, quarter-wave and half-wave retardance. These states can be easily transferred by rotating the wire grating. The performance of this device was achromatic over 0.1-0.7 THz. Then, they applied the $\mathrm{VO}_{2}$ film and grating structure into TIR geometry and further realized an active $\mathrm{THz}$ polarization controller (Figure $2 \mathrm{~d}$ ). This device shows an average modulation depth of $99.75 \%$ in the range of $0.2-1.1 \mathrm{THz}$ [27]. Unlike the former, the operation state of this device is controlled by the state of $\mathrm{VO}_{2}$, which is a phase-changed material. When $\mathrm{VO}_{2}$ was in insulating phase, the device worked as a linear rotator with an almost linear $p$-polarized output from $0.8-1.5 \mathrm{THz}$ (as shown in Figure 2e). While in the metallic phase, this device became a quarter-wave converter with a circular polarized output from $0.8-1.5 \mathrm{THz}$ (as shown in Figure 2f). Thus, the polarization state of the reflect wave is determined by both the grating structure and the conductivity of the interface. Similar active polarization may also be realized by using material with tunable conductivity. 


\section{THz Surface Plasmon Resonance Sensing and Devices Based on ATR}

\subsection{Surface Plasmon and Surface Plasmon Resonance}

Surface plasmon (SP) is a kind of collective excitation, which usually occurs at the interface between a conductor and a dielectric. This transverse magnetic mode propagates along the interface, and the field amplitude evanesces exponentially perpendicular to the interface. The surface plasmon polariton (SPP) will be excited by the collective oscillations of free charge carriers at a metal-dielectric interface provided the dielectric constant of the two media is opposite. The most attractive property of the SPP is that the field is confined in the vicinity of the surface. This resonance, usually called surface plasmon resonance (SPR), can be extremely sensitive to the slight changes in the refractive index of the surrounding media. Herein, plenty of research has been conducted for SPR sensing. Besides, studies have shown that graphene can also support well-confined SP modes at both MIR and $\mathrm{THz}$ regions. Gan [30] numerically investigated excitation of SP supported by doped graphene sheets at THz frequencies with ATR via Otto geometry (Figure 3a). To match the momentum between the highly confined plasmon modes and the incident radiation, the surface conductivity of graphene should be adjusted, such as varying doping levels or using few-layer graphene. Polyvinylidene fluoride (PVDF) is a kind of conducting polymer with efficient metal-like reflectance, which also supports $\mathrm{SP}$ modes at the $\mathrm{THz}$ region. Hassani et al. [26] realized $\mathrm{THz}$ plasmon-like excitation using PVDF covering a solid-core polymeric Bragg fiber via phase matching. Sensitivity of the SPR sensor using the angular interrogation method is defined as the ratio of the change in the resonance angle $\left(\theta_{S P R}\right)$ with the change in the refractive index $\left(n_{a}\right)$ of the sensing layer [42]:

$$
S_{n}=\frac{\delta \theta_{S P R}}{\delta n_{a}}
$$

(a)

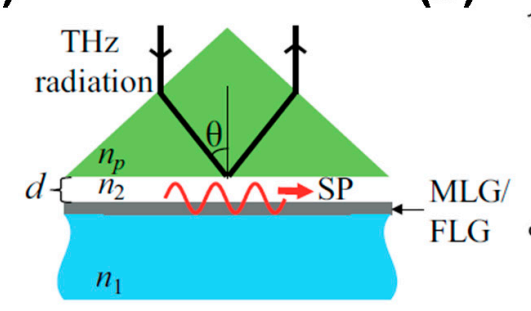

(b)

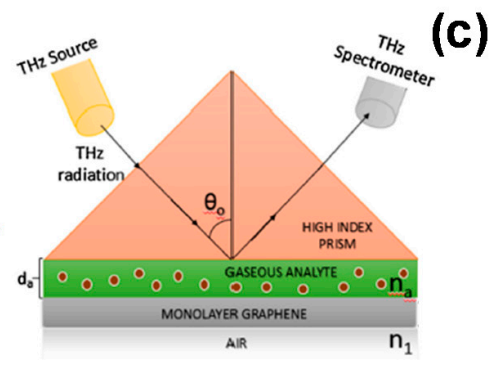

(c)

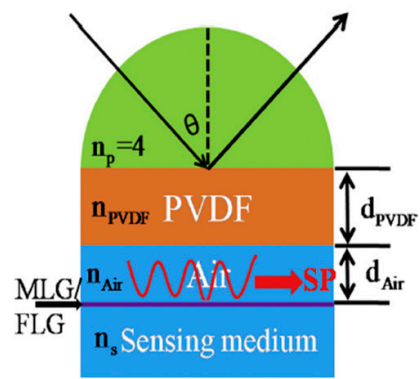

Figure 3. Surface plasmon sensor structure based on Otto or Kretschmann configuration. (a) Surface plasmon (SP) supported by doped graphene sheets at $\mathrm{THz}$ frequencies with attenuated total reflection (ATR) via the Otto geometry. Reproduced with permission from [30] (C) 2012 AIP Publishing. (b) SPR-based gas sensor in THz frequency with the Otto configuration. Reproduced with permission from [42] (C) 2016 Elsevier. (c) THz SPP excited on the dielectric-Polyvinylidene fluoride (PVDF) interface with prism coupling in the Kretschmann configuration. Reproduced with permission from [29] (C) 2018 Elsevier. $n$ : refractive index, $d$ : thickness, $\theta$ : incident angle, MLG: monolayer graphene, FLG: few-layer graphene, SP: surface plasmon.

Figure of merit (FOM) estimates the performance of the sensor and is defined as the ratio of sensitivity to the full width at half maxima (FWHM) of the SPR curve [42]:

$$
F O M=\frac{S_{n}}{F W H M}
$$

The following is advanced research around the SPR sensor based on Otto or Kretschmann geometry. 


\subsection{SPR Devices Based on Otto Geometry}

Purkayastha et al. [42] proposed an SPR-based gas sensor in $\mathrm{THz}$ frequency with Otto configuration using free standing doped monolayer graphene (Figure 3b). The proposed sensor could achieve an ultrahigh figure of merit about $1150 \mathrm{RIU}^{-1}$ (refractive index unit). It was found that the gap distance between the prism and graphene plays an important role in the coupling efficiency, which further determines the sensitivity and accuracy of the proposed sensor. However, the sample fabricating becomes a challenge since a tiny air gap needs to be maintained in this Otto geometry. To solve this problem, another SPR based gas sensor was proposed by using the different dielectric polymer spacing layer instead of the air gap with the similar structure [43]. This structure achieves the FOM of $741 \mathrm{RIU}^{-1}$ and is more suitable and convenient for practical sensing. Zhang et al. [44] further modified this structure and realized an active control SPR sensor through the magnetic field. The dielectric properties of graphene could be changed through the magnetic field, thus the sensitivity of the sensor could be optimized by properly adjusting the magnetic field. Another hybrid structure consisting of a double-layer graphene, a thin dielectric film, and planar waveguide was proposed, which can be used to support a surface plasmon polariton mode in long range [45]. This hybrid sensor structure could achieve sensitivity of $292 \mathrm{RIU}^{-1}$. The SPR sensors achieved the matching of the SPR mode between ATR geometry and graphene, and further confine the THz electric field in the vicinity of the surface.

\subsection{SPR Devices Based on Kretschmann Configuration}

The SPR coupling in the Kretschmann configuration was designed and numerically investigated [46]. In this structure, the SPP was excited on the interface between dielectric layer and PVDF. The sensitivity of the sensor was determined by the thickness of the PVDF and the sample. Another Kretschmann configuration (Figure 3c) that support two SPP modes coupling was invested [29]. The highest sensitivity of $730 \mathrm{RIU}^{-1}$ in gas detection can be obtained through this structure. The SPP excitation in this structure was mainly determined by the fermi energy level of graphene, thickness of PVDF and air (or coupling layer), and the layer number of graphene determines the coupling effects of the two SPP modes and finally affected the sensitivity. Both the Kretschmann and Otto configuration need to meet the momentum match condition, thus the effect of these two configurations are similar, with only differences in structures and materials between each other. The sensing methods above all are based on inducing $\mathrm{THz}$ waves with various incident angles at a certain frequency, which could be a challenge for their applications due to the optical path needed to be adjusted accordingly. Fixing the incident angle while using continuous-wave seems a better way to solve this problem.

\section{Spectroscopy Analysis}

\subsection{Dielectric Properties and Absorption Characteristics}

The interaction between $\mathrm{THz}$ wave and material could result in individual responses according to the chemical components and microstructure, thus making $\mathrm{THz}$ spectroscopy a powerful tool to analyze substances and detect responses. THz-ATR spectroscopy is very convenient and suitable for obtaining dielectric properties and absorption characteristics for the substance in the form of liquid, powder, or thin film. We concluded the samples with the parameter calculated in Table 1. All the measurements were taken under room temperature. The following are the reports on the dielectric properties and absorption characteristics of the testing substance. 
Table 1. The optical and dielectric characteristics of certain substance obtained by THz-ATR spectroscopy reported in the papers.

\begin{tabular}{|c|c|c|c|c|}
\hline Material & State & Region (THz) & Parameters & Reference \\
\hline Water & \multirow{9}{*}{ Liquid } & $0.1-1.6$ & $\alpha, n$ & [47] \\
\hline Water & & $0.3-3.6$ & ATR & [48] \\
\hline Water & & $0.5-12$ & $A$ & \multirow{2}{*}{ [49] } \\
\hline Aqueous glycine solution & & $0.5-12$ & $A$ & \\
\hline Methanol & & $0.3-3.6$ & ATR & \multirow{2}{*}[48]{} \\
\hline 2-Propanol & & $0.3-3.6$ & ATR & \\
\hline $\mathrm{NaCl}$ & & & & \multirow{3}{*}{$\begin{array}{l}{[50]} \\
{[32]}\end{array}$} \\
\hline $\mathrm{NaI}$ & & $0.2-1.1$ & $\alpha, n \alpha, n$ & \\
\hline L-(+)-tartaric acid & & $0.2-1.2$ & $\alpha, n$ & \\
\hline InAs & \multirow{17}{*}{ Solid } & $1.0-2.1$ & $\varepsilon$ & [47] \\
\hline Porcine tissues & & $0.01-1.00$ & $\varepsilon$ & [51] \\
\hline Pyridoxine (vitamin B6) & & & ATR & \multirow{7}{*}{ [48] } \\
\hline Riboflavin (vitamin B2) & & & & \\
\hline Thiamine hydrochloride (vitamin B1) & & & ATR & \\
\hline L-tartaric acid & & $0.3-3.6$ & ATR & \\
\hline D-mannitol & & & ATR & \\
\hline D-sorbitol & & & ATR & \\
\hline Xylitol & & & ATR & \\
\hline glycine powder & & $0.5-12$ & $A$ & \multirow[t]{4}{*}{ [49] } \\
\hline Azotetrazolate (explosive materials) & & & $A$ & \\
\hline Pentahydrate (explosive materials) & & & $A$ & \\
\hline $\begin{array}{l}\text { Diammonium (explosive materials) } \\
\text { azotetrazolate }\end{array}$ & & $0.2-3.2$ & $A$ & \\
\hline $\begin{array}{l}\text { Guanidinium azotetrazolate } \\
\text { (explosive materials) }\end{array}$ & & & $A$ & \\
\hline $\begin{array}{l}\text { Triaminoguanidinium azotetrazolate } \\
\text { (explosive materials) }\end{array}$ & & & $A$ & \\
\hline $\begin{array}{l}\text { Hexanitrohexaazaisowurtzitane } \\
\text { (explosive materials) }\end{array}$ & & & $A$ & \\
\hline $\begin{array}{l}\text { Tetraoxadinitroisowurtzitane } \\
\text { (explosive materials) }\end{array}$ & & & $A$ & \\
\hline
\end{tabular}

Hirori et al. [47] derived the dielectric functions of InAs and distilled liquid water through the measured ATR spectra. Sasaki et al. [51] obtained the dielectric properties of porcine tissues, including dermis, subcutaneous tissue, and muscle. Newnham et al. [48] studied the ATR spectra of nine kinds of crystalline solid materials and three kinds of liquids, including L-ascorbic acid (vitamin C), citric acid, L-tartaric acid, D-mannitol, D-sorbitol, xylitol, pyridoxine, riboflavin, thiamine hydrochloride, water methanol, and 2-propanol. All the solid materials showed distinct absorption peaks in the 0.3-3.6 THz region, which arise from low-frequency vibrations and phonon modes. Unlike the solid materials, the liquids, water methanol and 2-propanol, showed unstructured absorption. Ogawa et al. [49] obtained and analyzed the spectra of the aqueous glycine solution and glycine powder in the $0.5-12 \mathrm{THz}$ region. The differences of absorption peaks between powder and solution could be explained by that the low frequency vibrational mode in glycine molecules changed with the hydrogen bonding in water. Soltani et al. [50] promoted a method to monitor the crystallization of $\mathrm{NaCl}$ and $\mathrm{NaI}$ out of watery solution through the variation of the absorption coefficient and refractive index. Their absorption coefficient shows a similar decrease with the increase of the concentration, while the change in the refractive index is completely opposite. Based on this, crystallization of tartaric acid in the act was investigated [32]. Three different stages in the process of crystallization (supersaturated solution, nucleation stage, and final crystal) were changed in the relative contributions to the total volume, showing varied absorption character in each stage, which could be caught by ATR continuous measurements. The absorption characteristics of six prospective explosive materials 
by using transmission and ATR from 0.2 to about $3.2 \mathrm{THz}$ were investigated by Palka et al. [52]. The positions of peaks showed a reasonable consistence to the transmission results, which suggested the feasibility of ATR for identification of these materials. Figure 4 shows the absorption spectra of 4 kinds of them through the ATR method. Since the ATR geometry is very sensitive to the change of the interface, the contact between the sample, especially for powder and the ATR prism could also affect the results. Thus, there is difference between these two measurements. Besides, the penetration depth at lower frequency is bigger than that in the higher region, according to Equation (2), so the absorption characteristic could be more distinguishable at lower frequency in the $\mathrm{THz}$ region.
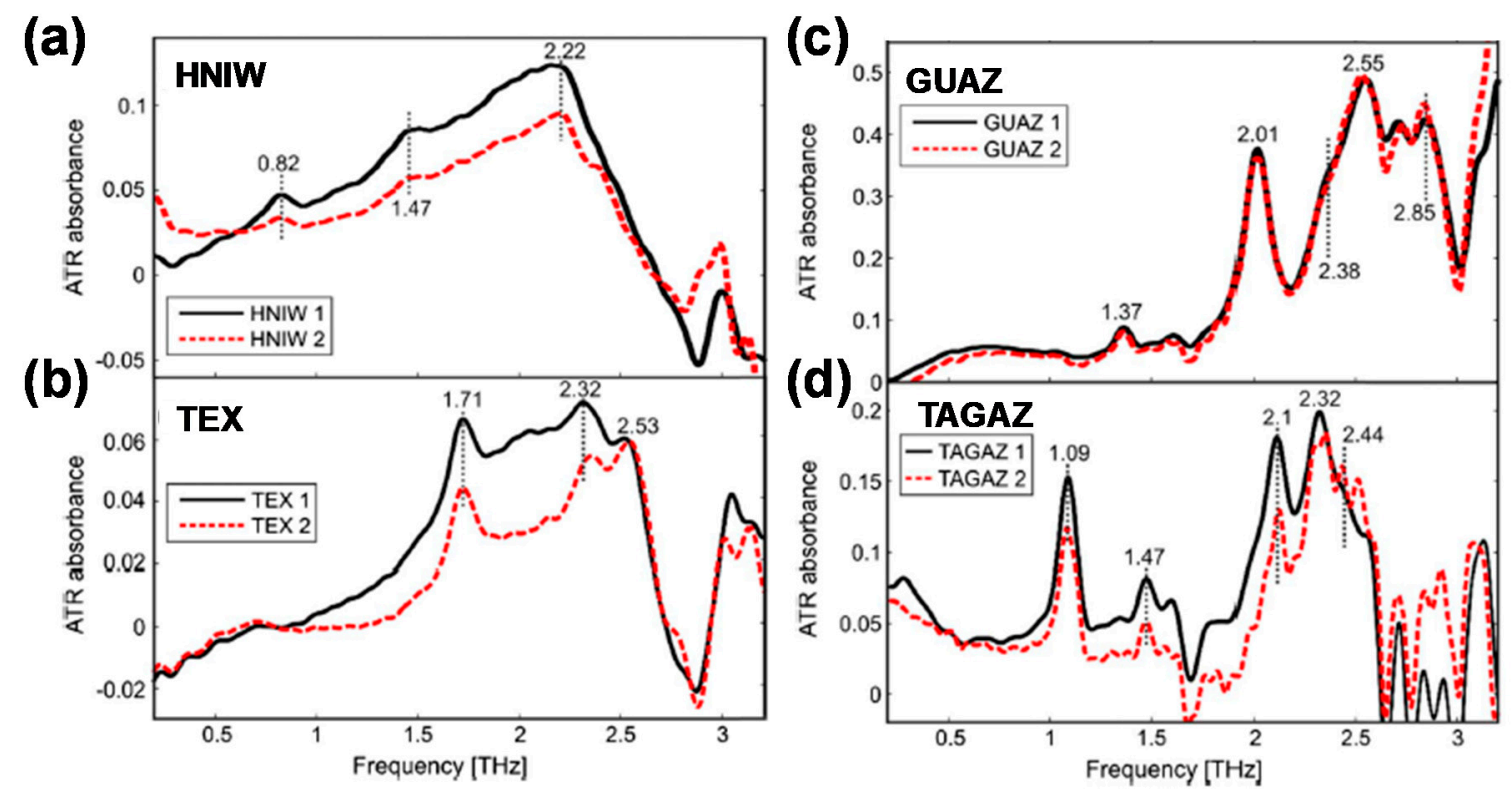

Figure 4. ATR absorbance of four explosives with distinguished character. (a) HNIW: Hexanitrohexaazaisowurtzitane, (b) TEX: Tetraoxadinitroisowurtzitane, (c) GUAZ: Guanidinium azotetrazolate, (d) TAGAZ: Triaminoguanidinium azotetrazolate. Reproduced with permission from [27], Copyright (C) 2018, The Optical Society.

\subsection{Hydrogen Bonds and Hydration State}

Hydrogen bond water network perturbations are referred to as hydration dynamics. The timescales for the dynamics of water molecules affected by the presence of a solute fall in the sub-picosecond region correspond to the THz region in the frequency domain. THz-ATR spectroscopy has been proved as a sensitive tool to analyze the water network in an aqueous solution. The absence of solute can affect the original dynamics of the surrounding water molecules and will contribute to the change of the dielectric spectra. For further qualitative and quantitative study of the response characteristic of aqueous solutions, analysis on hydrogen bonds and calculations around hydration were further carried out, with the absorption spectra and dielectric spectra as the following.

Hydrogen bonds have been demonstrated in various aqueous liquids. Roth et al. [53] found that they significantly influenced the Coulomb systems structure in the imidazolium-based ionic liquids. Nagai et al. [39] analyzed the dielectric dispersion of water in the THz region, indicating that the high-frequency vibrational modes should a make contribution to the dielectric dispersion. Then, Yada et al. [54,55] determined the dielectric character of water (Figure 5a) and its isotopes with different temperature, and decomposed them into four components: slow relaxation, fast relaxation at, intermolecular stretching vibration, and intermolecular libration. This research shows the unparalleled superiority of THz-ATR for evaluating the hydrogen-bonding system. 
(a)

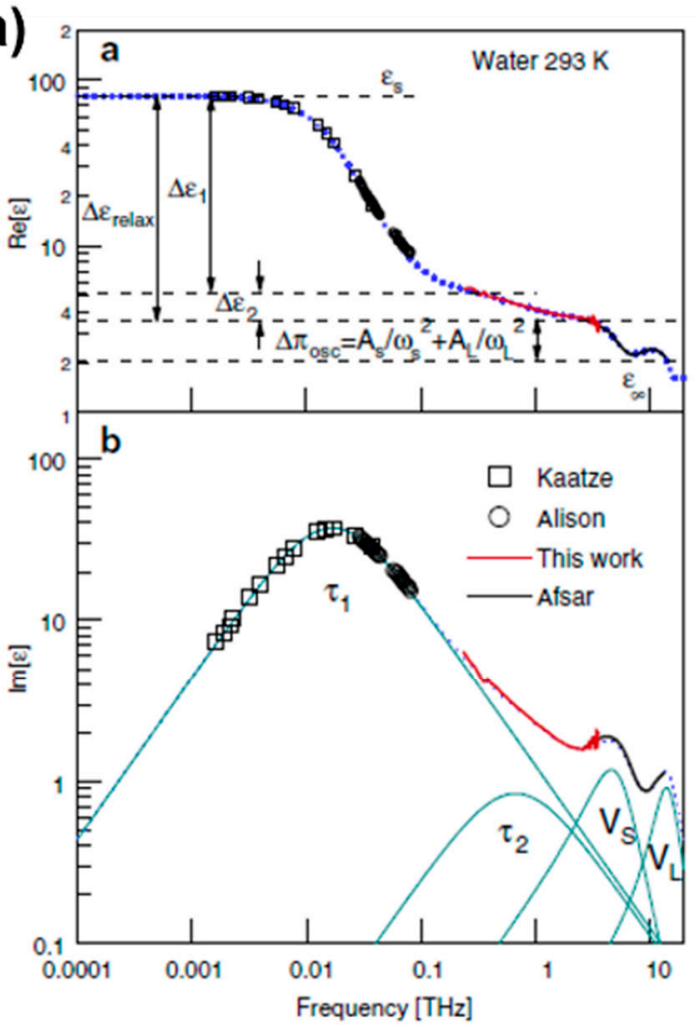

(b)

(c)
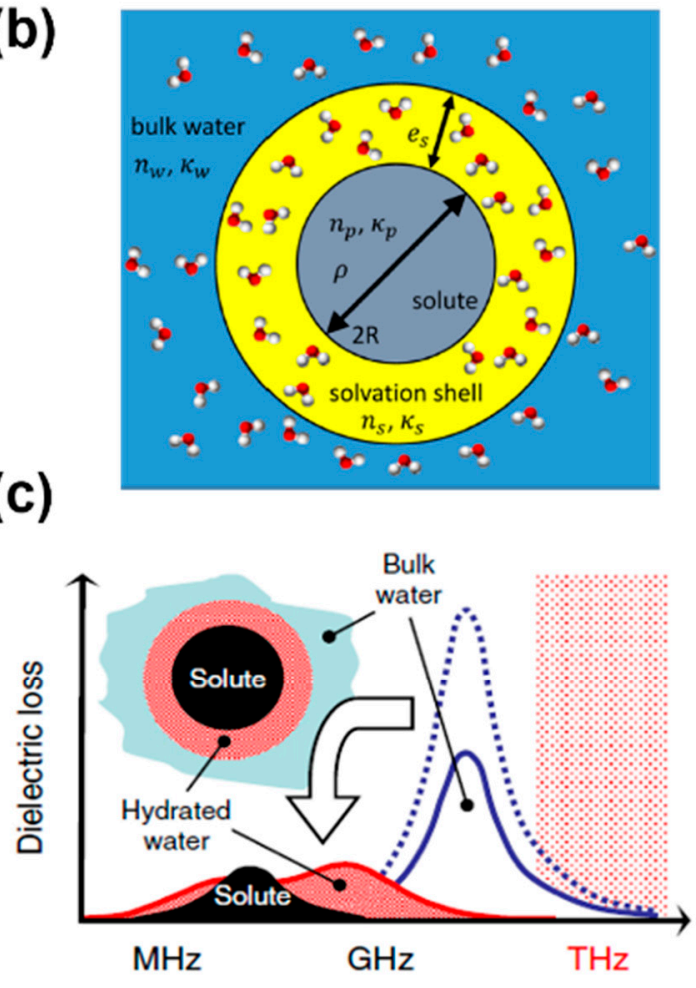

Figure 5. Explanation of dielectric response in the THz region for hydrogen bonds and hydration in aqueous solution. (a) The real part (up) and imaginary part (down) of the complex dielectric permittivity of the pure water. Reproduced with permission from [54], Copyright @ 2008, Elsevier. (b) Model of water molecules and solutes in aqueous, the water molecules are segmented into two stations, bulk water and solvation shell (hydrated water). Reproduced with permission from [56], Copyright (c) 2017, American Chemical Society. (c) Dielectric loss spectra of solute, hydrated water, and bulk water. Reproduced with permission from [57], Copyright (C) 2008, Elsevier.

Analysis on the hydration state is an attractive subject in exploring the structures and assessing the functions of biomolecules or chemical molecules in the aqueous matrix. Figure $5 \mathrm{~b}$ shows the simple model of water molecules and solutes in aqueous, the water molecules are segmented into two stations, bulk water and solvation shell (hydrated water). These components collectively contribute to the response of the dielectric loss spectra, as shown in Figure 5c. Based on these, the concentration-dependent hydration state in saccharide solutions was successfully evaluated [57-59], which further shows the strong relationship with the structure and groups of the saccharide molecule. For biomolecules, like phospholipid bilayers, amino acids, short peptides, and protein and carbohydrate polymers, their structure also are found to be related to the hydration state through the analysis on hydration numbers $[56,58,60,61]$. For chemical molecules, 2-butoxyethanol (2BE), shows a temperature-dependent relationship with the hydration number [31]. The hydration state of the surfactant was also found to be changed by the structural transitions [62]. Thus, the hydration state could be evaluated through calculating the hydration numbers from dielectric responses in the THz-ATR system.

\subsection{Component Analysis}

Based on the obtained characteristics in the $\mathrm{THz}$ region, qualitative and quantitative analysis methods could be used to analyze the components for practical samples. The convenience of the ATR configuration, such as non-invasion to the sample and without complex pre-treatment, makes it possible and suitable for the rapid detection and discrimination or even online monitoring. 
Naito et al. [63] analyzed the correlation between the absorption spectra of raw milk and the milk components by the partial least square regression and full cross validation as a test for the calibration models, the results showing that THz-ATR spectroscopy is suitable to predict several milk contents such as milk fat, total solid, and somatic cell counts. Cherkasova et al. [37] analyzed blood plasma specimens from healthy rats and diabetic rats. The results showed that spectra of blood plasma specimens obtained from diabetic rats had small but significant differences from those of healthy rats. Further, they studied the ATR spectra of palm skin in a consecutive measurement after glucose intake [64]. The results show a correlation between the changes in the blood glucose level and the variations of the ATR spectra, demonstrating the possibility of a non-invasive real-time measurement of blood glucose concentration. Takeya et al. [65] obtained a concentration dependence of ATR spectra with $\mathrm{NaCl}$ solutions and theophylline solutions. The concentration vibration only changed the amplitude of the ATR spectra other than the refractive index. Dohi et al. [66] applied THz-ATR spectroscopy to detect changes in the physical properties of lactose during the lubrication process required for drug formulation. They found that both the concentration ratio of components and blending time determined the final magnitude of spectra at the lactose-specific region. Liu et al. [67] obtained the absorption spectra of three kinds of honey, Medlar, Vitex, and Acacia. The apparent distinction among them could be attributed to the different types and contents of chemical compositions, such as glucose, fructose, and water. The quality of honey could be identified by the partial least squares-discriminant analysis (PLS-DA) model in the range of $0.5-1.5 \mathrm{THz}$, of which the accuracy of the validation set reached was $88.46 \%$. Soltani et al. [68] presented a THz-ATR setup and investigated the mixtures of water and ground calcium carbonate (GCC). Both refractive index and absorption coefficient showed correlation with the GGC concentration in the range between 30 and $40 \mathrm{wt} \%$, which proved the feasibility for the distinction of various concentrations of GCC in inline measurements. Qin et al. [69] determined the complex refractive indices of tetracycline hydrochloride $(\mathrm{TCH})$ in pure water and in pure milk at the 0.3-2.0 THz region. The presence of TCH could lower the complex refractive index at an equivalent frequency in the whole band, which could be related to the change in the relaxation dynamics of hydration water. The quantitative determination of $\mathrm{TCH}$ in pure water and in pure milk was further realized by analyzing the concentration dependence of complex refractive indices at $0.5 \mathrm{THz}$.

\subsection{In Situ Research for Biomaterials}

The picosecond dynamics of hydration, which could be relative to the characteristics of nucleic acid, protein, cell, and tissue, can be effectively evaluated through the dielectric properties in the $\mathrm{THz}$ region [70]. Besides, the THz wave is non-ionizing, which is safe for the biomedical samples. Thus, the THz-ATR system could be suitable for in situ research for biomaterials and biomedicine. Here, we reviewed research that mainly focuses on the cell samples.

Shiraga et al. [71,72] determined the complex dielectric constant of cultured human cancer cells (DLD-1, HEK293, and HeLa) in the THz region. They found that the dielectric responses below $1.0 \mathrm{THz}$ best characterize the particular water dynamics of cancer cells. Later in their research [59], the hydration state of an intact HeLa cell monolayer was investigated. Compared to the bulk water molecules, these intracellular hydration water molecules exhibited slowed down re-orientation dynamics relative to that of bulk water. Grognot et al. [73] proposed real-time measurements, which could monitor the cytoplasm leakage. The $\mathrm{THz}$ signal shows great sensitivity to the intracellular protein concentration. Zou et al. [74] also conducted a study on the cultured living human breast epithelial cells (MCF10A). The dielectric constant and dielectric loss of cells were determined, and their evolution under oxidative stress response was discussed. The above all demonstrated that THz-ATR spectroscopy could allow in situ, noninvasive, real-time, and rapid monitoring of living cells.

\section{Imaging Application}

ATR shows promise for use in an imaging mode to get precise absorption information without the inevitable noise induced by Fresnel refraction under general specular reflections $[33,75]$. Research 
shows THz-ATR imaging methods could ideally monitor and analyze liquid and biological samples. A typical THz-ATR imaging system is shown in Figure 6. The raster scanning method, point-by-point scanning by moving the prism in $y$ or $z$ direction, is used for obtaining image data of the samples.

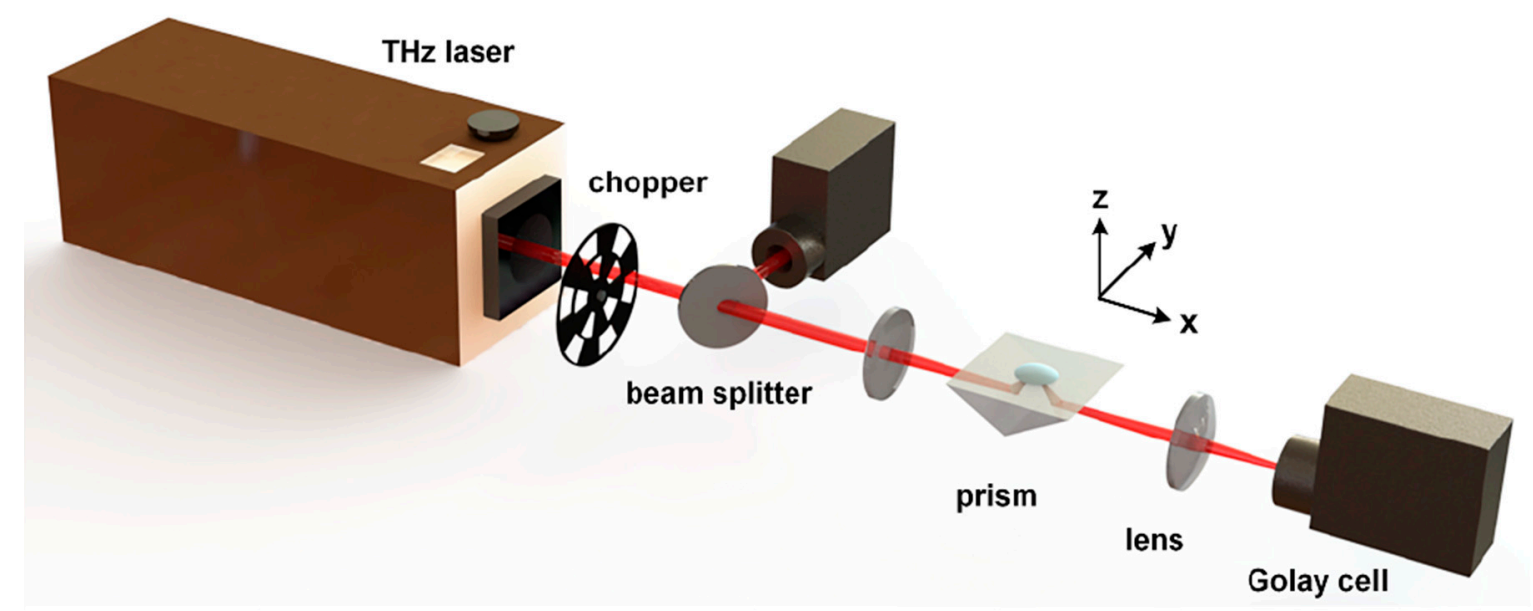

Figure 6. The setup of the vertically scanning imaging system. Reproduced with permission from [76], Copyright (C) 2018, The Optical Society.

Wojdyla et al. [77] presented a THz-ATR imaging technique, using the relative differential spectral phase of two orthogonal polarizations. This system showed advantages on the subwavelength ability in longitudinal resolution. Martinez-Meza et al. [78] used THz-ATR technology to study three most used skin-hydrants in commercial moisturizers and their interaction with the skin of a subject. Images of the forearm were obtained taking THz spectra images in sequential times. The results showed that the $\mathrm{THz}$ amplitude clearly decreased for all three substances between application and first measurement. Lanolin and hyaluronic acid approximately maintained the amplitude of the $\mathrm{THz}$ signal and glycerin slowly started to recover the reference state of the skin. Liu et al. [33] demonstrated an attenuated TIR imaging system. The surface information of the sample can be obtained by two-dimensionally scanning. The effective imaging area, image resolution, and polarization dependence of contrast enhancement and stability improvement were discussed in detail. The THz-ATR image information of distilled water (Figure 7a), solid agar (Figure 7b), and porcine tissue (Figure 7c) were obtained, showing high sensitivity for the proposed method. While comparing the results in Figure 7a, the image obtained by the $p$-polarized THz (right one) shows better contrasts than the elliptically polarized one (left one), thus indicating that polarization is one of the key factors for the quality of the image. In their following research [76], detailed analysis of the optimization for vertically scanning THz-ATR imaging has been presented. The optimum prism design was determined, considering the TIR condition, effective ATR imaging area, utilization of the prism, output heights of the reflected beams, and spatial resolution. They also found that the $p$-polarized waves can contribute to achieve minimum error. 


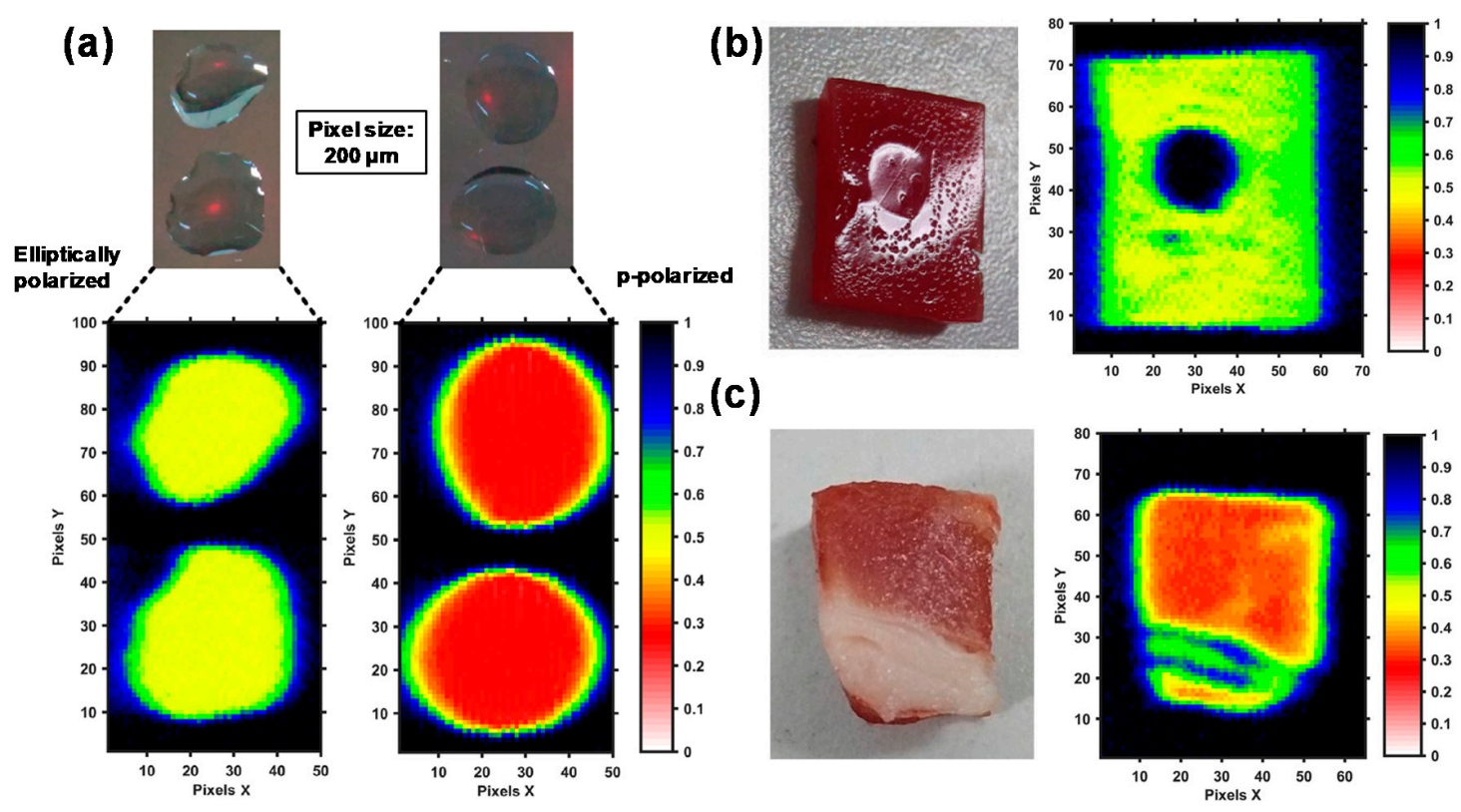

Figure 7. THz-ATR imaging of (a) distilled water, (b) a piece of blood agar, and (c) porcine tissue. Reproduced with permission from [33], Copyright (C) 2017, IOP Publishing.

\section{Conclusions and Perspectives}

Here, we introduce the basic optical geometry of the ATR system and summed up the factors that influence the system. High-performance, broadband, active, and multi-function modulators based on ATR have been proposed and discussed, showing attractive potentiality and unrivalled advantages in amplitude and polarization modulation. Surface plasmon sensing methods based on ATR configuration, Otto and Kretschmann, have been proposed and theoretically validated the feasibility, which will open a new window for high performance plasmonic sensor for gas, liquid, and so forth. The advantage of the ATR method makes it convenient for charactering liquid, powder, and thin-film materials, especially for the surface layer characteristics, which will be further developed in the rapid and noninvasive method for both spectroscopy and imaging. Due to the effective control of intensive absorption of water, the research of solute and water molecules around, including molecular dynamics, hydrogen bonds, and hydration in aqueous solution, can be realized through ATR geometry. In particular, the biomaterial-related picosecond dynamics of hydration lies in the $\mathrm{THz}$ band, making it suitable to obtain the characteristics of nucleic acid, protein, cell, and tissue for further application in biomedicine. To conclude, ATR in the THz region shows unique strengths as following:

(1) Ultra-sensitivity to the conductive interface makes ATR an ideal platform for modulation; active modulation would also be realized by materials with tunable conductive properties.

(2) The evanescent field supported by ATR offers great opportunity for realizing SPR with specific materials and structures which support the SPP mode.

(3) In situ research for biomaterials and biomedicine can be convenient and effective through ATR geometry.

Despite ATR being applied as an interesting platform in THz modulation, sensing, spectroscopy and imaging, the limitations also make it challenging for further application. The performance of modulation and SP sensing with ATR geometry is based on the properties of materials, such as graphene and PVDF and configuration of the device. Thus, the fabrication of this modulator and sensor remains the biggest challenge for achieving high performance. For spectroscopy and imaging, the ambiguous response mechanism limits the applications, especially when it comes to both complex external and internal situations for samples. In addition, the influence factors, such as difference in 
environment and preparation for the samples, can affect the results substantially. Based on these, further research could be developed from these aspects:

(1) For modulation and sensing, the performance is determined by the fabrication, thus attention on the uniformity, repeatability, and stability of graphene, PVDF, and other tunable conductive layers should be paid. Especially for SPR sensing, a tiny difference from thickness and properties in each layer even would make it invalid.

(2) Present SPR sensing based on ATR could be a challenge for their application due to the optical path needed to be adjusted accordingly. Fixing the incident angle while using continuous-wave could be a better way to solve this problem.

(3) Spectra characteristics of the material should be further mined, which could be realized by some advanced algorithms such as deep learning. Besides, theoretical explanations for the spectroscopy response in the $\mathrm{THz}$ region also need to be further supplemented and completed. A simulation study, such as molecular dynamic and vibration, could offer guidance.

(4) Reducing environmental disturbance and standardizing the test process are in great need for reliable spectroscopy and imaging results. The ambient temperature should be stabilized in a certain range and humidity should be controlled as low as possible. For thin-films and powder samples, the pressure, which was usually applied on the sample in order to make the sample and the ATR prism surface contact well, can also affect the result and should be optimized.

In summary, the ATR technique has broadened the scope of research for $\mathrm{THz}$ spectroscopy and imaging, and it is expected to provide more extensive applications in the future.

Author Contributions: Y.H. and L.X. searched and classified the literature; Y.H., L.X. and Y.Y. wrote the manuscript. R.S. polished the manuscript. All authors have read and agreed to the published version of the manuscript.

Funding: This work was founded by the Natural Science Foundation of Zhejiang Province for Distinguished Young Scholars (LR18C130001).

Acknowledgments: The authors gratefully acknowledge the financial support provided by the Natural Science Foundation of Zhejiang Province for Distinguished Young Scholars (LR18C130001).

Conflicts of Interest: The authors declare no conflict of interest.

\section{References}

1. Mathanker, S.K.; Weckler, P.R.; Wang, N. Terahertz (THz) applications in food and agriculture: A review. Trans. ASABE 2013, 56, 1213-1226.

2. Smith, R.M.; Arnold, M.A. Terahertz time-domain spectroscopy of solid samples: Principles, applications, and challenges. Appl. Spectrosc. Rev. 2011, 46, 636-679.

3. Yang, X.; Zhao, X.; Yang, K.; Liu, Y.; Liu, Y.; Fu, W.; Luo, Y. Biomedical applications of terahertz spectroscopy and imaging. Trends Biotechnol. 2016, 34, 810-824.

4. Qin, J.Y.; Ying, Y.B.; Xie, L.J. The detection of agricultural products and food using terahertz spectroscopy: A review. Appl. Spectrosc. Rev. 2013, 48, 439-457.

5. Shen, Y.C. Terahertz pulsed spectroscopy and imaging for pharmaceutical applications: A review. Int. J. Pharm. 2011, 417, 48-60.

6. Naftaly, M.; Molloy, J.F.; Magnusson, B.; Andreev, Y.M.; Lanskii, G.V. Silicon carbide-a high-transparency nonlinear material for THz applications. Opt. Express 2016, 24, 2590-2595.

7. Wang, Z.F.; Liu, F. Self-assembled Si (111) surface states: 2D Dirac material for THz plasmonics. Phys. Rev. Lett. 2015, 115, 026803.

8. Nagatsuma, T.; Ducournau, G.; Renaud, C.C. Advances in terahertz communications accelerated by photonics. Nat. Photonics 2016, 10, 371-379.

9. Li, Z.; Guan, L.; Li, C.X.; Radwan, A. A secure intelligent spectrum control strategy for future THz mobile heterogeneous networks. IEEE Commun. Mag. 2018, 56, 116-123.

10. Fan, K.B.; Suen, J.Y.; Liu, X.Y.; Padilla, W.J. All-dielectric metasurface absorbers for uncooled terahertz imaging. Optica 2017, 4, 601-604. 
11. Stantchev, R.I.; Sun, B.Q.; Hornett, S.M.; Hobson, P.A.; Gibson, G.M.; Padgett, M.J.; Hendry, E. Noninvasive, near-field terahertz imaging of hidden objects using a single-pixel detector. Sci. Adv. 2016, 2, e1600190.

12. Yamaguchi, S.; Fukushi, Y.; Kubota, O.; Itsuji, T.; Ouchi, T.; Yamamoto, S. Brain tumor imaging of rat fresh tissue using terahertz spectroscopy. Sci. Rep. 2016, 6, 30124.

13. Sizov, F.F. Infrared and terahertz in biomedicine. Semicond. Phys. Quantum Electron. Optoelectron. 2017, 20, 273-283.

14. Panchenko, A.; Tyndyk, M.; Smolyanskaya, O.; Sulatskiy, M.; Kravtsenyuk, O.; Balbekin, N.; Khodzitsky, M. THz spectroscopy of whole blood, plasma and cells in mice of SHR line with various pathology. Int. Conf. Young Sci. Spec. Opt. 2016, 735, 012081.

15. Sommer, S.; Raidt, T.; Fischer, B.M.; Katzenberg, F.; Tiller, J.C.; Koch, M. THz-spectroscopy on high density polyethylene with different crystallinity. J. Infrared Millim. Terahertz Waves 2016, 37, 189-197.

16. Garcia-Wernersson, J.; Rodilla, H.; Diaz-Bolado, A.; Dahlback, R.; Gradinarsky, L.; Josefson, M.; Stake, J. Non-destructive density measurements of pharmaceutical products by $\mathrm{THz}$ imaging. In Proceedings of the 2016 41st International Conference on Infrared, Millimeter, and Terahertz Waves (IRMMW-THz), Copenhagen, Denmark, 25-30 September 2016.

17. Hindle, F.; Kuuliala, L.; Mouelhi, M.; Cuisset, A.; Bray, C.; Vanwolleghem, M.; Devlieghere, F.; Mouret, G.; Bocquet, R. Monitoring of food spoilage by high resolution THz analysis. Analyst 2018, 143, 5536-5544.

18. Shin, H.J.; Choi, S.W.; Ok, G. Qualitative identification of food materials by complex refractive index mapping in the terahertz range. Food Chem. 2018, 245, 282-288.

19. Qin, J.Y.; Xie, L.J.; Ying, Y.B. Feasibility of terahertz time-domain spectroscopy to detect tetracyclines hydrochloride in infant milk powder. Anal. Chem. 2014, 86, 11750-11757.

20. Xie, L.J.; Yao, Y.; Ying, Y.B. The application of terahertz spectroscopy to protein detection: A review. Appl. Spectrosc. Rev. 2014, 49, 448-461.

21. Xu, W.D.; Xie, L.J.; Ye, Z.Z.; Gao, W.L.; Yao, Y.; Chen, M.; Qin, J.Y.; Ying, Y.B. Discrimination of transgenic rice containing the Cry1 Ab protein using terahertz spectroscopy and chemometrics. Sci. Rep. 2015, 5, 11115.

22. Wang, C.; Qin, J.Y.; Xu, W.D.; Chen, M.; Xie, L.J.; Ying, Y.B. Terahertz imaging applications in agriculture and food engineering: A review. Trans. Asabe 2018, 61, 411-424.

23. Sun, Y.W.; Degl'Innocenti, R.; Ritchie, D.A.; Beere, H.E.; Xiao, L.; Ruggiero, M.; Zeitler, J.A.; Stantchev, R.I.; Chen, D.N.; Peng, Z.C.; et al. Graphene-loaded metal wire grating for deep and broadband THz modulation in total internal reflection geometry. Photonics Res. 2018, 6, 1151-1157.

24. Liu, X.D.; Chen, X.Q.; Parrott EP, J.; Pickwell-Macpherson, E. Exploiting a metal wire grating in total internal reflection geometry to achieve achromatic polarization conversion. Photonics Res. 2017, 5, 299-304.

25. Endo, T.; Kerman, K.; Nagatani, N.; Takamura, Y.; Tamiya, E. Label-free detection of peptide nucleic acid-DNA hybridization using localized surface plasmon resonance based optical biosensor. Anal. Chem. 2005, 77, 6976-6984.

26. Hassani, A.; Dupuis, A.; Skorobogatiy, M. Surface-plasmon-resonance-like fiber-based sensor at terahertz frequencies. J. Opt. Soc. Am. B-Opt. Phys. 2008, 25, 1771-1775.

27. Liu, X.; Chen, X.; Parrott, E.P.; Han, C.; Humbert, G.; Crunteanu, A.; Pickwell-MacPherson, E. Invited Article: An active terahertz polarization converter employing vanadium dioxide and a metal wire grating in total internal reflection geometry. APL Photonics 2018, 3, 051604.

28. Liu, X.D.; Chen, Z.F.; Parrott EP, J.; Ung BS, Y.; Xu, J.B.; Pickwell-MacPherson, E. Graphene based terahertz light modulator in total internal reflection geometry. Adv. Opt. Mater. 2017, 5, 1600697.

29. Zhu, J.Q.; Ruan, B.X.; You, Q.; Guo, J.; Dai, X.Y.; Xiang, Y.J. Terahertz imaging sensor based on the strong coupling of surface plasmon polaritons between PVDF and graphene. Sens. Actuators B-Chem. 2018, 264, 398-403.

30. Gan, C.H. Analysis of surface plasmon excitation at terahertz frequencies with highly doped graphene sheets via attenuated total reflection. Appl. Phys. Lett. 2012, 101, 111609.

31. Arikawa, T.; Nagai, M.; Tanaka, K. Hydration structures of 2-butoxyethanol monomer and micelle in solution. Chem. Phys. Lett. 2009, 477, 95-101.

32. Soltani, A.; Gebauer, D.; Duschek, L.; Fischer, B.M.; Colfen, H.; Koch, M. Crystallization caught in the act with terahertz spectroscopy: Non-classical pathway for L-(+)-tartaric acid. Chem. A Eur. J. 2017, 23, 14128-14132. 
33. Liu, H.X.; Wang, Y.Y.; Xu, D.G.; Wu, L.M.; Yan, C.; Yan, D.X.; Tang, L.H.; He, Y.X.; Feng, H.; Yao, J.Q. High-sensitivity attenuated total internal reflection continuous-wave terahertz imaging. J. Phys. D Appl. Phys. 2017, 50, 375103.

34. Shiraga, K.; Ogawa, Y.; Kondo, N.; Irisawa, A.; Imamura, M. Evaluation of the hydration state of saccharides using terahertz time-domain attenuated total reflection spectroscopy. Food Chem. 2013, 140, 315-320.

35. Soltani, A.; Jahn, D.; Duschek, L.; Castro-Camus, E.; Koch, M.; Withayachumnankul, W. Attenuated total reflection terahertz time-domain spectroscopy: Uncertainty analysis and reduction scheme. IEEE Trans. Terahertz Sci. Technol. 2016, 6, 32-39.

36. Soltani, A.; Probst, T.; Busch, S.F.; Schwerdtfeger, M.; Castro-Camus, E.; Koch, M. Error from delay drift in terahertz attenuated total reflection spectroscopy. J. Infrared Millim. Terahertz Waves 2014, 35, 468-477.

37. Cherkasova, O.P.; Nazarov, M.M.; Angeluts, A.A.; Shkurinov, A.P. Analysis of blood plasma at terahertz frequencies. Opt. Spectrosc. 2016, 120, 50-57.

38. Nakanishi, A.; Kawada, Y.; Yasuda, T.; Akiyama, K.; Takahashi, H. Terahertz time domain attenuated total reflection spectroscopy with an integrated prism system. Rev. Sci. Instrum. 2012, 83, 033103.

39. Nagai, M.; Yada, H.; Arikawa, T.; Tanaka, K. Terahertz time-domain attenuated total reflection spectroscopy in water and biological solution. Int. J. Infrared Millim. Waves 2006, 27, 505-515.

40. Harada, Y.; Ukhtary, M.S.; Wang, M.J.; Srinivasan, S.K.; Hasdeo, E.H.; Nugraha AR, T.; Noe, G.T.; Sakai, Y.; Vajtai, R.; Vajtai, R.; et al. Giant terahertz-wave absorption by monolayer graphene in a total internal reflection geometry. ACS Photonics 2017, 4, 121-126.

41. Li, J.S. Switching terahertz wave with grating-coupled Kretschmann configuration. Opt. Express 2017, 25, 19422-19428.

42. Purkayastha, A.; Srivastava, T.; Jha, R. Ultrasensitive THz-plasmonics gaseous sensor using doped graphene. Sens. Actuators B-Chem. 2016, 227, 291-295.

43. Srivastava, T.; Purkayastha, A.; Jha, R. Graphene based surface plasmon resonance gas sensor for terahertz. Opt. Quantum Electron. 2016, 48, 334.

44. Zhang, Z.X.; Su, H.; Gong, H.B.; Wang, S.X.; Zhang, M.; Liang, H.W.; Zhang, C. Graphene based surface plasmon resonance gas sensor with magnetic field control for terahertz. In Proceedings of the 2017 Progress in Electromagnetics Research Symposium-Fall (Piers-Fall), Singapore, 19-22 November 2017; pp. 3068-3072.

45. Ruan, B.X.; You, Q.; Zhu, J.Q.; Wu, L.M.; Guo, J.; Dai, X.Y.; Xiang, Y.J. Terahertz biochemical sensor based on strong coupling between waveguide mode and surface plasmons of double-layer graphene. IEEE Sens. J. 2018, 18, 7436-7441.

46. Li, X.J.; Song, J.; Zhang, J.X.J. Integrated terahertz surface plasmon resonance on polyvinylidene fluoride layer for the profiling of fluid reflectance spectra. Plasmonics 2016, 11, 1093-1100.

47. Hirori, H.; Yamashita, K.; Nagai, M.; Tanaka, K. Attenuated total reflection spectroscopy in time domain using terahertz coherent pulses. Jpn. J. Appl. Phys. Part 2-Lett. Express Lett. 2004, 43, L1287-L1289.

48. Newnham, D.A.; Taday, P.F. Pulsed terahertz attenuated total reflection spectroscopy. Appl. Spectrosc. 2008, 62, 394-398.

49. Ogawa, Y.; Cheng, L.; Hayashi, S.; Fukunaga, K. Attenuated total reflection spectra of aqueous glycine in the terahertz region. IEICE Electron. Express 2009, 6, 117-121.

50. Soltani, A.; Stubling, E.M.; Koch, M. Monitoring the crystallization of $\mathrm{NaCl}$ and NaI with THz ATR Spectroscopy. In Proceedings of the 2015 40th International Conference on Infrared, Millimeter and Terahertz Waves (IRMMW-THz), Hong Kong, China, 23-28 August 2015.

51. Sasaki, K.; Mizuno, M.; Wake, K.; Watanabe, S. Monte Carlo simulations of skin exposure to electromagnetic field from $10 \mathrm{GHz}$ to $1 \mathrm{THz}$. Phys. Med. Biol. 2017, 62, 6993.

52. Palka, N.; Szala, M.; Czerwinska, E. Characterization of prospective explosive materials using terahertz time-domain spectroscopy. Appl. Opt. 2016, 55, 4575-4583.

53. Roth, C.; Peppel, T.; Fumino, K.; Kockerling, M.; Ludwig, R. The importance of hydrogen bonds for the structure of ionic liquids: Single-crystal X-ray diffraction and transmission and attenuated total reflection spectroscopy in the terahertz region. Angew. Chem. 2010, 49, 10221-10224.

54. Yada, H.; Nagai, M.; Tanaka, K. Origin of the fast relaxation component of water and heavy water revealed by terahertz time-domain attenuated total reflection spectroscopy. Chem. Phys. Lett. 2008, 464, 166-170.

55. Yada, H.; Nagai, M.; Tanaka, K. The intermolecular stretching vibration mode in water isotopes investigated with broadband terahertz time-domain spectroscopy. Chem. Phys. Lett. 2009, 473, 279-283. 
56. Grognot, M.; Gallot, G. Relative contributions of core protein and solvation shell in the terahertz dielectric properties of protein solutions. J. Phys. Chem. B 2017, 121, 9508-9512.

57. Arikawa, T.; Nagai, M.; Tanaka, K. Characterizing hydration state in solution using terahertz time-domain attenuated total reflection spectroscopy. Chem. Phys. Lett. 2008, 457, 12-17.

58. Huang, H.C.; Liu, Q.; Zhu, L.G.; Li, Z.R. Characterizing the hydration state of L-threonine in solution using terahertz time-domain attenuated total reflection spectroscopy. In Proceedings of the 2017 International Conference on Optical Instruments and Technology-IRMMW-THz Technologies and Their Applications, Beijing, China, 28-30 October 2017; Volume 10623.

59. Shiraga, K.; Suzuki, T.; Kondo, N.; De Baerdemaeker, J.; Ogawa, Y. Quantitative characterization of hydration state and destructuring effect of monosaccharides and disaccharides on water hydrogen bond network. Carbohydr. Res. 2015, 406, 46-54.

60. Hishida, M.; Tanaka, K. Long-range hydration effect of lipid membrane studied by terahertz time-domain spectroscopy. Phys. Rev. Lett. 2011, 106, 158102.

61. Morales-Hernandez, J.A.; Singh, A.K.; Villanueva-Rodriguez, S.J.; Castro-Camus, E. Hydration shells of carbohydrate polymers studied by calorimetry and terahertz spectroscopy. Food Chem. 2019, 291, 94-100.

62. Hishida, M.; Tanaka, K. Transition of the hydration state of a surfactant accompanying structural transitions of self-assembled aggregates. J. Phys. Condens. Matter 2012, 24, 284113.

63. Naito, H.; Ogawa, Y.; Shiraga, K.; Kondo, N.; Hirai, T.; Osaka, I.; Kubota, A. Inspection of milk components by terahertz attenuated total reflectance (THz-ATR) spectrometer equipped temperature controller. In Proceedings of the 2011 IEEE/SICE International Symposium on System Integration (SII), Kyoto, Japan, 20-22 December 2011.

64. Cherkasova, O.; Nazarov, M.; Shkurinov, A. Noninvasive blood glucose monitoring in the terahertz frequency range. Opt. Quantum Electron. 2016, 48, 217.

65. Takeya, K.; Muto, K.; Ishihara, Y.; Kawase, K. Monitoring theophylline concentrations in saline using terahertz ATR spectroscopy. Appl. Sci. 2016, 6, 72.

66. Dohi, M.; Momose, W.; Yamashita, K.; Hakomori, T.; Sato, S.; Noguchi, S.; Terada, K. Application of terahertz attenuated total reflection spectroscopy to detect changes in the physical properties of lactose during the lubrication process required for drug formulation. Chem. Pharm. Bull. 2017, 65, 186-193.

67. Liu, W.; Zhang, Y.Y.; Yang, S.; Han, D.H. Terahertz time-domain attenuated total reflection spectroscopy applied to the rapid discrimination of the botanical origin of honeys. Spectrochim. Acta Part A Mol. Biomol. Spectrosc. 2018, 196, 123-130.

68. Soltani, A.; Busch, S.F.; Plew, P.; Balzer, J.C.; Koch, M. THz ATR spectroscopy for inline monitoring of highly absorbing liquids. J. Infrared Millim. Terahertz Waves 2016, 37, 1001-1006.

69. Qin, J.Y.; Xie, L.J.; Ying, Y.B. Rapid analysis of tetracycline hydrochloride solution by attenuated total reflection terahertz time-domain spectroscopy. Food Chem. 2017, 224, 262-269.

70. Sun, Q.; He, Y.; Liu, K.; Fan, S.; Parrott, E.P.; Pickwell-MacPherson, E. Recent advances in terahertz technology for biomedical applications. Quant. Imaging Med. Surg. 2017, 7, 345.

71. Shiraga, K.; Ogawa, Y.; Suzuki, T.; Kondo, N.; Irisawa, A.; Imamura, M. Determination of the complex dielectric constant of an epithelial cell monolayer in the terahertz region. Appl. Phys. Lett. 2013, 102, 053702.

72. Shiraga, K.; Ogawa, Y.; Suzuki, T.; Kondo, N.; Irisawa, A.; Imamura, M. Characterization of dielectric responses of human cancer cells in the terahertz region. J. Infrared Millim. Terahertz Waves 2014, 35, 493-502.

73. Grognot, M.; Gallot, G. Quantitative measurement of permeabilization of living cells by terahertz attenuated total reflection. Appl. Phys. Lett. 2015, 107, 103702.

74. Zou, Y.; Liu, Q.; Yang, X.; Huang, H.C.; Li, J.; Du, L.H.; Li, Z.R.; Zhao, J.H.; Zhu, L.G. Label-free monitoring of cell death induced by oxidative stress in living human cells using terahertz ATR spectroscopy. BioMed. Opt. Express 2018, 9, 14-24.

75. Liu, H.X.; Wang, Y.Y.; Xu, D.G.; Duan, P.; Nie, M.T.; Shi, J.; Yan, C.; He, Y.X.; Chen, T.N.; Feng, H.; et al. Attenuated total internal reflection imaging with continuous terahertz wave. In Proceedings of the 2016 41st International Conference on Infrared, Millimeter, and Terahertz Waves (IRMMW-THz), Copenhagen, Denmark, 25-30 September 2016.

76. Liu, H.X.; Wang, Y.Y.; Xu, D.G.; Jiang, Z.N.; Li, J.N.; Wu, L.M.; Yan, C.; Tang, L.H.; He, Y.X.; Yan, D.X.; et al. Optimization for vertically scanning terahertz attenuated total reflection imaging. Opt. Express 2018, 26, 20744-20757. 
77. Wojdyla, A.; Gallot, G. Attenuated internal reflection terahertz imaging. Opt. Lett. 2013, 38, 112-114.

78. Martinez-Meza, L.H.; Rojas-Landeros, S.C.; Castro-Camus, E.; Alfaro-Gomez, M. Evaluation of skin moisturizer effects using terahertz time domain imaging. In Proceedings of the Photonics in Dermatology and Plastic Surgery 2018, San Francisco, CA, USA, 27 January-1 February 2018; Volume 10467.

(C) 2020 by the authors. Licensee MDPI, Basel, Switzerland. This article is an open access article distributed under the terms and conditions of the Creative Commons Attribution (CC BY) license (http://creativecommons.org/licenses/by/4.0/). 